\title{
Aortic-vertebral interaction in ankylosing spondylitis: syndesmophyte development at the juxta-aortic vertebral rim
}

\author{
Sovira Tan, ${ }^{1}$ Abhijit Dasgupta, ${ }^{1}$ John A Flynn, ${ }^{2}$ Michael M Ward ${ }^{\oplus}$
}

\begin{abstract}
Handling editor Josef S Smolen

- Additional material is published online only. To view please visit the journal online (http://dx.doi.org/10.1136/ annrheumdis-2018-214675).

${ }^{1}$ Intramural Research Program, National Institute of Arthritis and Musculoskeletal and Skin Diseases, National Institutes of Health, Bethesda, Maryland, USA

${ }^{2}$ University of Chicago Medical Center, Chicago, Illinois, USA
\end{abstract}

\section{Correspondence to}

Dr Michael M Ward, National Institutes of Health, Bethesda, MD 20892, USA;

wardm1@mail.nih.gov

Received 31 October 2018 Revised 26 February 2019 Accepted 23 March 2019 Published Online First 6 April 2019

\section{Check for updates}

(C) Author(s) (or their employer(s)) 2019. No commercial re-use. See rights and permissions. Published by BMJ.

To cite: $\operatorname{Tan} S$

Dasgupta A, Flynn JA,

et al. Ann Rheum Dis

2019:78:922-928

\section{ABSTRACT}

Objectives The aorta inhibits paravertebral ossification in diffuse idiopathic skeletal hyperostosis. We investigated if syndesmophytes in ankylosing spondylitis (AS) occurred less often at the vertebral rim near the aorta.

Methods We performed thoracolumbar CT scans in 60 subjects in this cross-sectional study. The midthoracic spine was also scanned in 22 subjects. We divided the rim of each intervertebral disc space (IDS) into 72 angular sectors, each of $5^{\circ}$. We computed syndesmophyte height in each sector, and the distance from the sector to the aorta. We evaluated if syndesmophyte size or frequency in a sector was associated with its distance from the aorta.

Results In the $180^{\circ}$ region of the vertebral rim centered on the sector closest to the aorta, syndesmophyte height and/or frequency varied with the distance of the sector to the aorta, with the lowest frequency and smallest mean syndesmophyte height at the sector along the rim nearest the aorta. Additionally, syndesmophytes were less common in subjects and at IDSs where the aorta was anatomically closer to the vertebra. No syndesmophytes were present in the sector closest to the aorta in subjects whose aorta-vertebral distance was less than $2 \mathrm{~mm}$, but syndesmophytes were progressively more common among subjects whose aortas lay further from the rim. Conclusions Syndesmophytes occurred less commonly and were smaller at the thoracolumbar vertebral rim near the aorta. These findings suggest that mechanical factors extrinsic to the spine and not solely vertebral inflammation, influence syndesmophyte development in AS.

In ankylosing spondylitis (AS), spinal fusion can result from the development of syndesmophytes that join adjacent vertebral bodies, and from fusion of the zygapophyseal joints. ${ }^{1-3}$ Syndesmophytes are thought to develop in response to local inflammation in the enthesis at the annulus-bone junction or in the subcortical vertebral body, but the processes that govern syndesmophyte development are incompletely understood. ${ }^{4-6}$ Vertebral osteitis and fat metaplasia, detected by MRI, is associated with future development of syndesmophytes at the adjacent vertebral rim, but this association has only modest sensitivity and specificity. ${ }^{78}$ Clinical factors other than inflammation that may influence the development of syndesmophytes have not been widely considered.

Emerging evidence suggests that mechanical stress may modify syndesmophyte development and their distribution. ${ }^{9}$ Patients with certain

\section{Key messages}

What is already known about this subject?

- Paravertebral ossification in DISH occurs less commonly on the left side of the spine.

- Whether the aorta is associated with the local presence or absence of syndesmophytes in ankylosing spondylitis has not been investigated.

What does this study add?

- Using CT scans, we show that syndesmophytes are less frequent and smaller in the area of the vertebral rim adjacent to the aorta than in neighbouring regions in the lower thoracic and upper lumbar spine.

How might this impact on clinical practice or future developments?

- Local inhibition of syndesmophyte growth may be due to mechanical effects of aortic pulsations.

- Factors other than vertebral inflammation can influence syndesmophyte formation in ankylosing spondylitis.

physically demanding occupations, particularly those with exposure to whole body vibration, have more extensive syndesmophytes than those with less active occupations. ${ }^{10}$ In the lower thoracic and upper lumbar vertebrae, syndesmophytes commonly develop at the posterolateral vertebral rim, which is the portion of the vertebral body typically subjected to the most mechanical stress. ${ }^{11}$ In contrast, syndesmophytes are less likely to develop at the anterior vertebral rim. The distribution of syndesmophytes within a disc space may provide clues to their pathogenesis.

Considering the anatomic proximity of the aorta to the vertebral rim, we hypothesised that the aorta might influence syndesmophyte development at the neighbouring region of the vertebrae. In diffuse idiopathic skeletal hyperostosis (DISH), paravertebral ossification in the thoracic spine preferentially develops contralateral to the aorta, as demonstrated by cases with situs inversus. ${ }^{12-14}$ This inhibition of paravertebral ossification has been attributed to the effects of constant aortic pulsation on the adjacent soft tissue. ${ }^{12} 15$ We hypothesised that similar inhibition due to extrinsic mechanical forces from the aorta may occur in AS. We investigated the 


\begin{tabular}{|c|c|c|}
\hline & Value & Range \\
\hline Age, years & $46.4 \pm 10.9$ & $24-69$ \\
\hline Men & $47(87)$ & \\
\hline Duration of AS, years & $19.0 \pm 11.4$ & $2-53$ \\
\hline HLA-B27 present & $40(85)^{*}$ & \\
\hline Bath AS Functional Index (0-100) & $27.5 \pm 23.4$ & $0-89.0$ \\
\hline Bath AS Disease Activity Index (0-10) & $2.9 \pm 2.0$ & $0-7.3$ \\
\hline Peripheral arthritis & $7(13)$ & \\
\hline Hip or shoulder involvement & $6(11)$ & \\
\hline Median Lumbar modified Stoke AS Spine score (0-36) & 4 & $0-26$ \\
\hline Radiographic lumbar syndesmophytes & $41(76)$ & \\
\hline
\end{tabular}

Plus-minus values are mean $\pm \mathrm{SD}$; other values are number (per cent).

${ }^{*}$ Of 47 subjects tested.

AS, ankylosing spondylitis.

association between the aorta and the local presence of syndesmophytes in the thoracolumbar spine in patients with AS, using the precise localisation provided by CT. Our research question was whether, in an affected intervertebral disc space (IDS), syndesmophytes were more likely or less likely to be present at
T10-T11
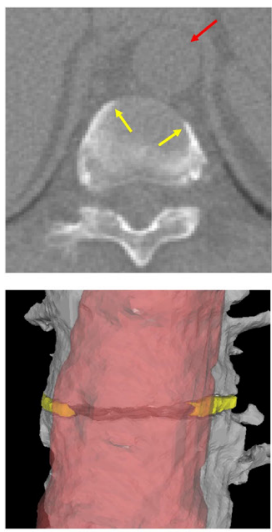

L1-L2
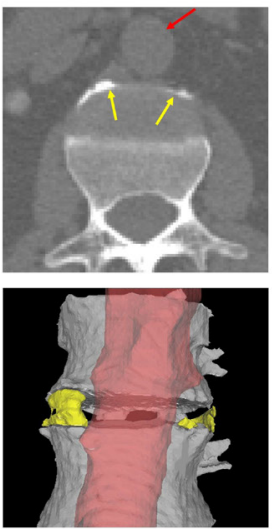

T11-T12
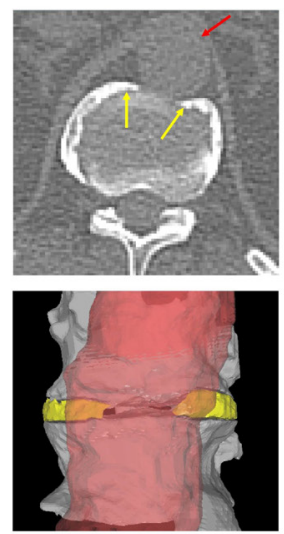

L2-L3
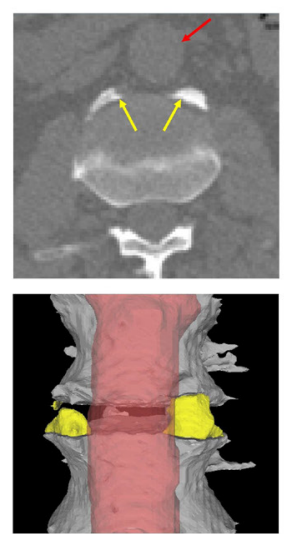

T12-L1
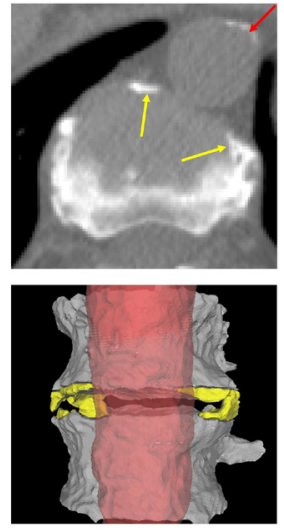

L3-L4
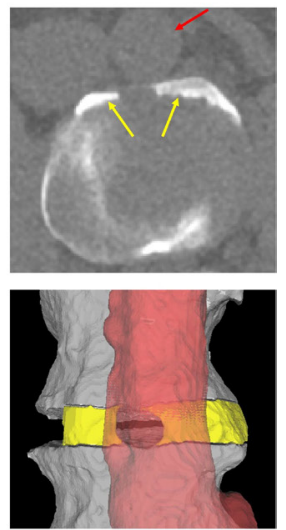

Figure 1 Examples of intervertebral disc spaces (from T10-T11 to $\mathrm{L} 3-\mathrm{L} 4$ ) where the portion of the rim closest to the aorta is free of syndesmophyte but is surrounded by syndesmophytes on both sides. The first row shows an axial slice of the CT scan, with yellow arrows pointing to syndesmophytes and the red arrow pointing to the aorta. The second row shows the corresponding three-dimensional surface reconstructions, with yellow surfaces denoting syndesmophytes and semitransparent red showing the aorta. Each of the six examples is from a different subject. the vertebral rim closest to the aorta compared with other areas of the rim.

\section{METHODS}

\section{Patients and study protocol}

We invited adults age 18 or older with AS who were attending our clinics to participate in a research study that used spinal CT to quantitate syndesmophyte growth. ${ }^{11}$ All subjects fulfilled the modified New York criteria for the classification of AS and had a Bath AS Radiology Index (BASRI) lumbar spine score of 0 to 3 (ie, not completely fused) at study entry. ${ }^{16} 17$ Subjects with radiographic syndesmophytes were preferentially enrolled, as these were most informative. All subjects completed a physical examination, health questionnaires, laboratory tests, spine radiographs and spinal CT at study entry. Some subjects were followed prospectively, while others participated in substudies that required only a single evaluation. In this cross-sectional analysis, we included only data from the baseline visit. The study protocol was approved by the institutional review boards, and all subjects gave written informed consent.

\section{CT scanning and image analysis}

Sixty subjects had CT scans of the lower thoracic and lumbar spine, which provided data on six IDS: T10-T11, T11-T12, T12-L1, L1-L2, L2-L3 and L3-L4. Four subjects were scanned too low and did not contribute data on T10-T11. The 22 most-recently enrolled subjects also had CT scans of the mid-thoracic spine. ${ }^{18}$ These subjects contributed data on five additional IDSs: T5-T6, T6-T7, T7-T8, T8-T9 and T9-T10. Therefore, 22 subjects had scans of 11 IDSs, 34 subjects had scans of only 6 IDSs and 4 subjects had scans of only 5 IDSs. Earlier scans were done using either a Philips Brilliance 64 (slice thickness $1.5 \mathrm{~mm}$ ) or GE Lightspeed Ultra (slice thickness $1.25 \mathrm{~mm}$ ) scanner, while more recent scans were done using Siemens Somatom Flash or Somatom Force (slice thickness $1.0 \mathrm{~mm}$ ) scanners. The estimated absorbed radiation dose was $8.01 \mathrm{mSv}$.

We computed syndesmophyte height around the vertebral rim using a validated semiautomated method. ${ }^{19}$ At each IDS, the $360^{\circ}$ of the entire vertebral rim was divided into 72 angular sectors of $5^{\circ}$ each. In each angular sector, height was computed for both ascending and descending syndesmophytes, which were summed and normalised to the local IDS height so that bridging had a value of 1.0, values between 0 and 1.0 represented the proportion of the IDS spanned by syndesmophytes and 0 indicated the absence of syndesmophytes. This measure is both reliable and valid. ${ }^{19} 20$

We next segmented the aorta, and computed the distance of each vertebral angular sector at a given IDS to the aorta at that level. Distance was calculated to the portion of the aortic wall closest to the rim, taken as the average distance from the superior and inferior rims. For each IDS, we identified the angular sector closest to the aorta, which we termed the abutting sector, and defined a $180^{\circ}$ region around this sector (ie, $90^{\circ}$ on either side) as the region for analysis (online supplementary figure 1). We used an IDS-specific region rather than a fixed anatomic region because the aorta migrates medially as it descends. ${ }^{21} \mathrm{We}$ computed syndesmophyte heights in this semicircle of angular sectors and included those subjects who had a syndesmophyte in at least one of these 37 angular sectors in the analysis of a given IDS. These subjects had syndesmophytes in the region near the aorta and therefore would provide an adequate test of associations with the aorta. Subjects who did not have syndesmophytes at a given IDS or who only had syndesmophytes far 


\section{A}

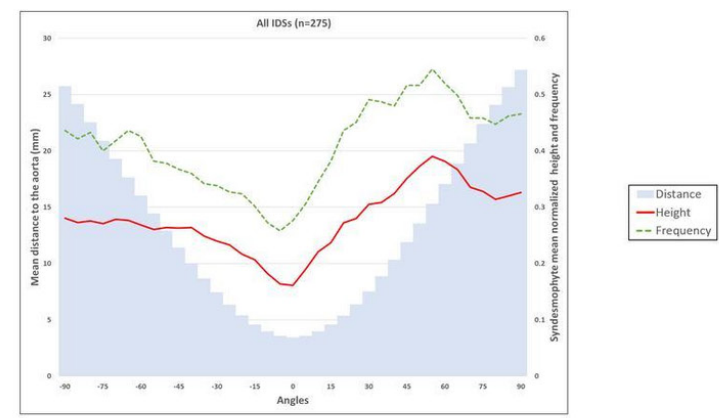

B

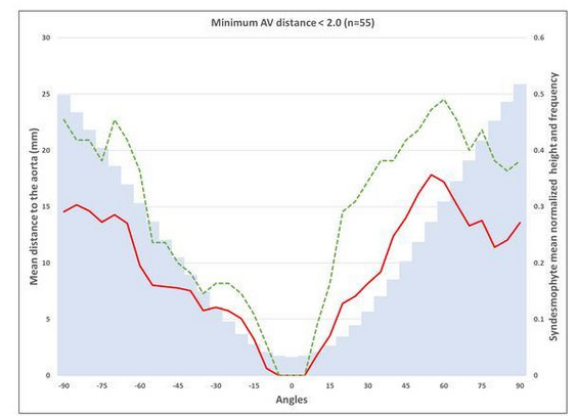

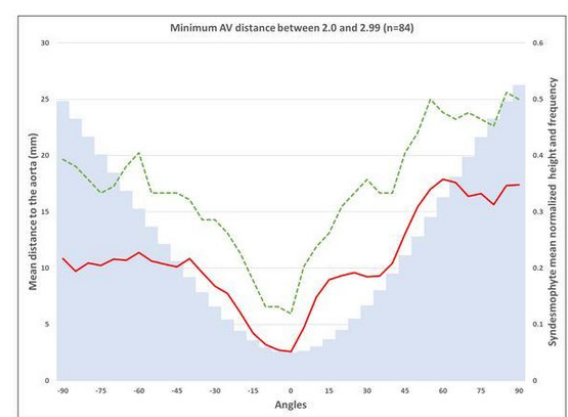

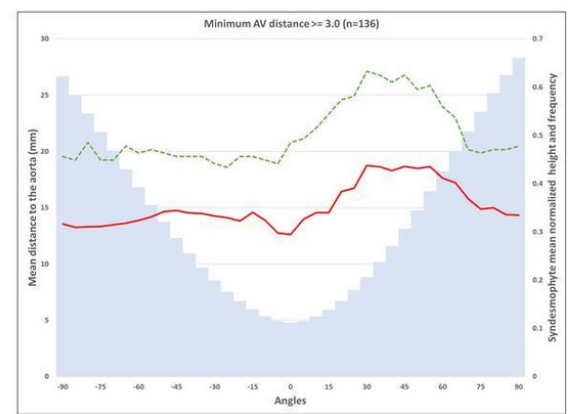

Figure 2 (A) Relationship between syndesmophytes and the distance of angular sectors to the aorta among all intervertebral disc spaces. The $\mathrm{X}$ axis represents the location of the angular sectors on the vertebral rim in relationship to the aorta. Plots are centered around the abutting sector. Angular sectors $90^{\circ}$ on either side of the abutting sector were analysed. The blue bars represent the mean distance of each angular sector to the closest part of the aortic wall, in millimetres (left $Y$ axis). Syndesmophyte frequency (green line) and mean syndesmophyte height (red line) are plotted for each angular sector (right $\mathrm{Y}$ axis). (B) Relationship stratified by distance of the aorta to the abutting sector (minimum aortovertebral (AV) distance $<2.0$ $\mathrm{mm}, 2.0-2.99 \mathrm{~mm}$ or $\geq 3.0 \mathrm{~mm}$ ). IDS, intervertebral disc space.

from the aorta (ie, further than $90^{\circ}$ away in either direction) would not be informative. Different subjects could therefore contribute data for different IDSs, depending on the location of their syndesmophytes.

Aortic wall calcifications and vertebral flattening were evaluated to examine whether local inflammatory or mechanical mechanisms might underlie the aortic-vertebral association. We noted the presence and location of aortic wall calcifications by visual inspection of the CT scans to determine if these were associated with syndesmophytes at the adjacent vertebral rim. We also visually examined the scans for evidence of flattening of the contour of the vertebral body by the aorta. ${ }^{22}$ Flattening of the vertebral centrum is an anatomic feature attributed to pressure on the bone from the adjacent aorta and was used as another indicator of mechanical aortic-vertebral interactions. ${ }^{23}$ Flattening was assessed qualitatively by one reader (MMW) as a left-right asymmetry in the contour of the anterior vertebral rim, with either a depression or straightening of the rim on the left side.

\section{Statistical analysis}

We analysed the aortic-syndesmophyte association in relation to three factors: the angular sectors (ie, position in the arc along the rim), the IDS level and the distance of the aorta to the abutting sector (hereafter, minAV distance). To investigate if the angular sectors close the aorta were less likely to have syndesmophytes than other nearby sectors, we first plotted syndesmophyte frequency (presence or absence of syndesmophyte at any angular sector) and mean syndesmophyte height in each angular sector in relation to the distance of the sector to the aorta. We then stratified the plots by IDS, and by minAV distance (in three groups: $<2.0 \mathrm{~mm}, 2.0-2.99 \mathrm{~mm}$ or $\geq 3.0 \mathrm{~mm}$ ). We also examined the frequency of syndesmophytes in the abutting sector as a function of the minAV distance at each IDS level.
We tested whether the presence of syndesmophytes in a given angular sector was associated with its location on the vertebral rim (ie, from $-90^{\circ}$ to $+90^{\circ}$ from the abutting sector), the minAV distance or IDS level, using a multivariable logistic regression model. We also tested the interaction between the position of the angular sector on the rim and the minAV distance because the plots suggested a dependence on the distance from aorta to rim. The model was implemented using generalised estimating equations (GEE) with an exchangeable correlation structure to account for multiple IDSs per patient. We computed robust SEs using sandwich estimators. To provide global estimates of the associations, we first represented the angular sector as a continuous variable. To provide detailed estimates of the interaction effects, we next modelled angular sector as a categorical variable. Results represent the relative odds of a syndesmophyte in a given angular sector. We also examined if local aortic wall calcification was associated with the presence of syndesmophytes by comparing model results in IDSs with or without adjacent aortic calcification. $\mathrm{R}$ programs were used for analysis.

\section{RESULTS}

Of the 60 subjects enrolled, 54 subjects had syndesmophytes on CT in the $180^{\circ}$ region of the vertebral rim neighbouring the aorta in at least one IDS and were included in the analysis. Most subjects were middle-aged men with long-standing AS (table 1). The number of subjects that contributed data to the analysis of a given IDS ranged from 9 subjects at T9-T10 to 42 subjects at T11-T12. The angular sector closest to the aorta at T5-T6 was at the left lateral aspect of the vertebral body, while at L3-L4 it was near the midline (online supplementary figure 2). Characteristics of subjects excluded from analysis are provided in online supplementary table 1.

Inspection of axial CT images revealed a relative absence of syndesmophytes at the vertebral rim adjacent to the aorta. In 


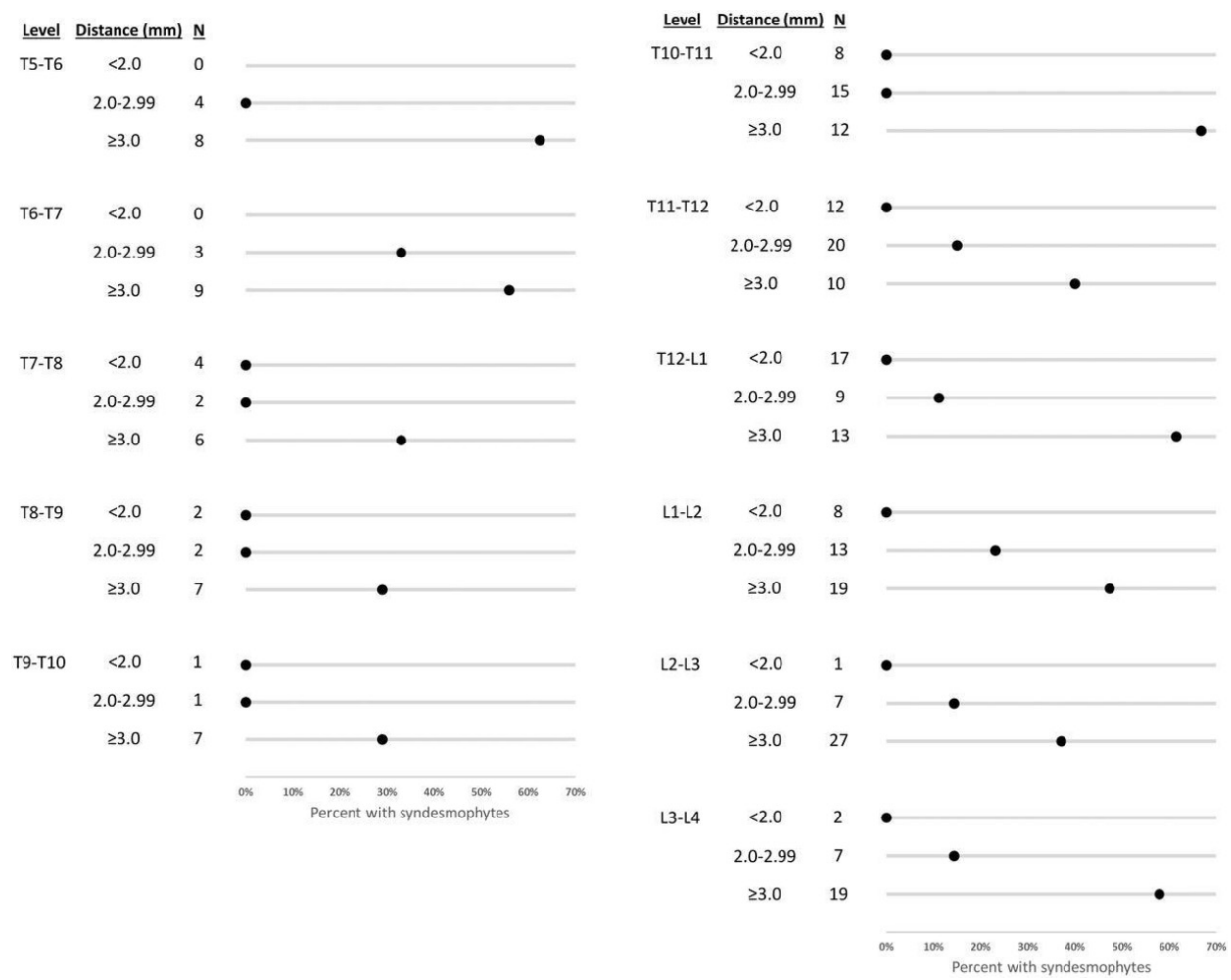

Figure 3 Association between syndesmophyte frequency in the abutting sector and the absolute distance (in millimetres) from the vertebral rim to the aorta by intervertebral disc space. The number $(\mathrm{N})$ of subjects that contributed data varied among the different intervertebral disc spaces.

several cases, syndesmophytes bracketed the region of the rim closest to the aorta (figure 1).

In plots of the association between the presence of syndesmophytes in a particular angular sector and the distance of the sector from the aorta, syndesmophyte frequency was lowest at the abutting sector and progressively increased at sectors along the rim further from the aorta (figure 2A). Mean syndesmophyte height had a similar pattern. Individual subject data showing syndesmophytes and their location relative to the aorta as heatmaps are presented in online supplementary figure 3 .

When individual IDSs were examined, the association was strongest at T10-T11, T11-T12, T12-L1 and L1-L2, and somewhat less pronounced at T7-T8, T8-T9 and L2-L3 (online supplementary figure 4). The minAV distance varied among IDSs, with means $<3 \mathrm{~mm}$ at the thoracolumbar junction, but $>4 \mathrm{~mm}$ for other IDSs (online supplementary figure 5). This pattern suggested that the differences across IDSs were mediated by how far the aorta was from the vertebra at different levels. There was a marked difference in the association by minAV distance. Syndesmophytes were not present near the abutting sector when minAV distance was $<2.0 \mathrm{~mm}$, while there was no association when minAV distance was $\geq 3.0 \mathrm{~mm}$ (figure $2 \mathrm{~B}$ ). The minAV distance also varied considerably among subjects. For example, at T11-T12, this distance ranged from $0.89 \mathrm{~mm}$ to $6.11 \mathrm{~mm}$ among different subjects. There was a graded association between the frequency of syndesmophytes and the minAV distance (figure 3). Syndesmophytes occurred less often near the aorta in those subjects whose aorta was closer to the vertebral rim. No syndesmophytes were present in the abutting sector in patients whose aorta was $<2 \mathrm{~mm}$ from the vertebral rim.

In the GEE model, both the location of the angular sector on the rim in relation to the aorta $(\mathrm{p}<0.0001)$ and minAV distance $(p<0.0001)$ were associated with the likelihood of a syndesmophyte at a given angular sector (online supplementary table 2).
There was also a significant interaction between these measures $(p<0.0001)$, such that the association of minAV distance was strongest at the abutting sector and nearby sectors. At the abutting sector, the odds of a syndesmophyte decreased by one-half with each millimetre decrease in minAV distance. However, at sectors more than $45^{\circ}$ from the abutting sector, there was no association between syndesmophytes and minAV distance (figure 4 and online supplementary table 3). IDS level was not associated with the presence of syndesmophytes (online supplementary tables 2 and 3).

Aortic wall calcification was present in 25 patients, mostly below L2, and only 11 patients had calcifications in the posterior aortic wall near an IDS. Associations between minAV distance and angular sector were similar in IDSs with and without adjacent aortic calcification (online supplementary figure 6).

Flattening of the left anterior vertebral rim was assessed for all vertebrae regardless of the presence of syndesmophytes. Flattening was observed primarily at the thoracolumbar junction, affecting $32 \%$ of T10 vertebrae, 37\% of T11 vertebrae, $50 \%$ of T12 vertebrae and $20 \%$ of L1 vertebrae, with rare or no involvement at other levels (figure 5). Flattening was present in $17 \%$ of vertebrae with minAV distance $<3.0 \mathrm{~mm}$, compared with $5.8 \%$ with $\operatorname{minAV}$ distance $\geq 3.0 \mathrm{~mm}$.

\section{DISCUSSION}

Understanding the processes that regulate the development of syndesmophytes could lead to the discovery of treatments to slow or stop spinal fusion. Studies to date have focused on spinal inflammation as the precursor of syndesmophytes. ${ }^{6-8}$ Our results indicate that factors extrinsic to the spine also influence the presence of syndesmophytes in AS. Syndesmophytes occurred less commonly and were smaller at the vertebral rim near the aorta, suggesting an inhibition of syndesmophyte growth. This 

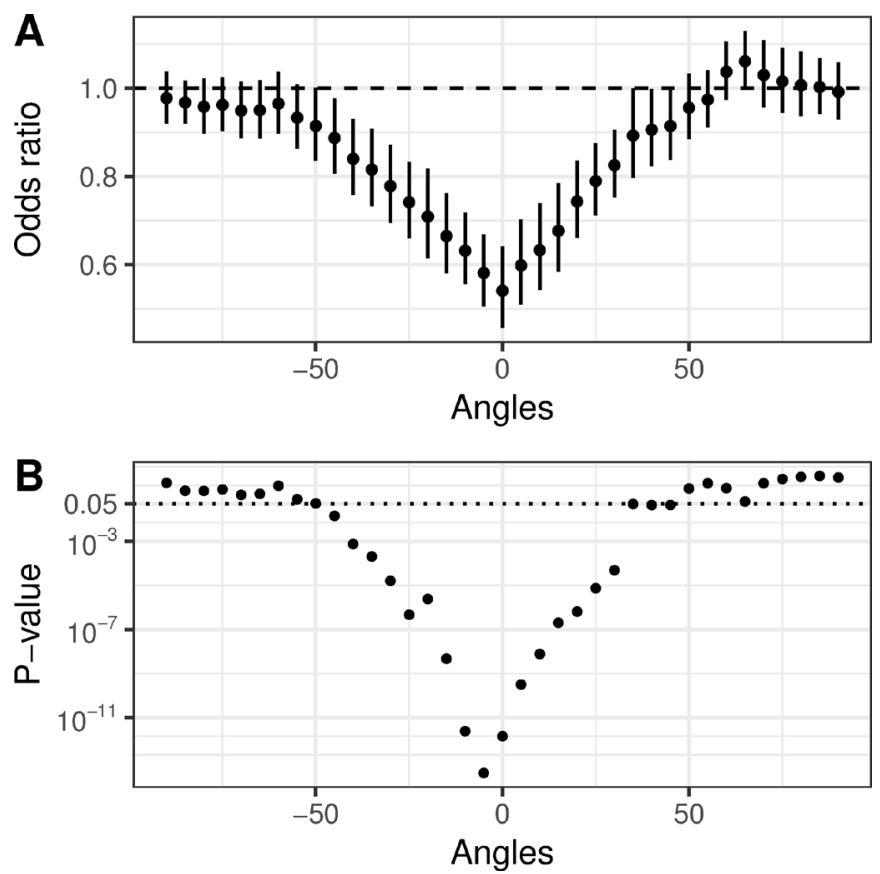

Figure 4 (A) Adjusted ORs of the association between minAV distance and presence of a syndesmophyte in an angular sector. The OR presents the likelihood for every $1 \mathrm{~mm}$ closer that the aorta is to the vertebral rim. At the abutting sector (labelled 0 ), for each $1 \mathrm{~mm}$ closer the aorta is to the rim, the odds that a syndesmophyte was present decreased by one-half. For angular sectors more distant from the abutting sector, the association of syndesmophytes with the distance of the aorta from the vertebral rim was less strong and was not significant at sectors more than $45^{\circ}$ away from the abutting sector. Error bars represent $95 \% \mathrm{Cls}$. This is a graphic representation of the model results provided in online supplementary table 3. (B) Corresponding $\mathrm{p}$ values for each OR.

association was stronger in those subjects whose aorta was closer to the spine, supporting a 'dose-response' association.

It is well known that paravertebral ossification in DISH mainly develops contralateral to the aorta. ${ }^{12-14}$ Ossification in DISH is easily visible on plain radiographs, which undoubtedly facilitated detection of its predominance on the right side of the spine. It is less well known that vertebral osteophytes also display laterality. In both imaging studies and studies of anatomic specimens, osteophytes in the thoracic spine mostly develop on the right side of the vertebral bodies. ${ }^{24-28}$ This laterality is not present in the lumbar spine inferior to the aortic bifurcation, suggesting that the aorta acts to inhibit osteophyte development on left side of the thoracic spine. ${ }^{25}$ Our findings related to syndesmophytes were quite similar, notwithstanding the inflammatory nature of AS. These similarities suggest that common processes may influence the development of both syndesmophytes and vertebral osteophytes. ${ }^{29}$ The small size of syndesmophytes relative to the ossification in DISH, and the very local effects of the aorta, likely helped conceal this association.

The inhibition of paravertebral bone growth in DISH and of vertebral osteophytosis has been attributed to the mechanical effects of aortic pulsations on the vertebrae and surrounding tissue. $^{12} 1524$ While this is also the most likely explanation for our observations in AS, direct testing of this hypothesis is not possible. It is not completely understood how mechanical effects inhibiting bone growth are transduced at the molecular level. Mechanisms other than mechanical effects, such as paracrine effects of substances released by vascular or perivascular
Not Flattened

Flattened

T10
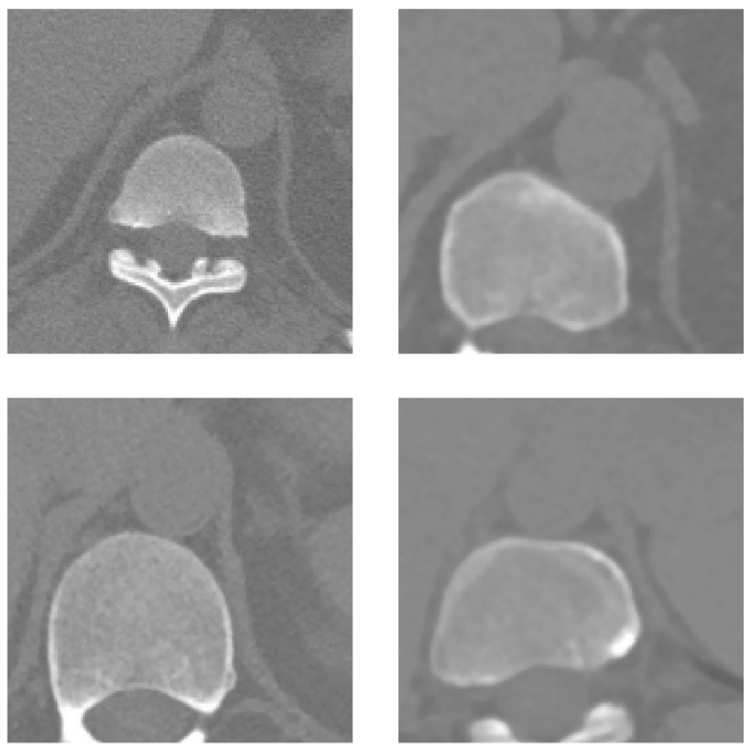

T11
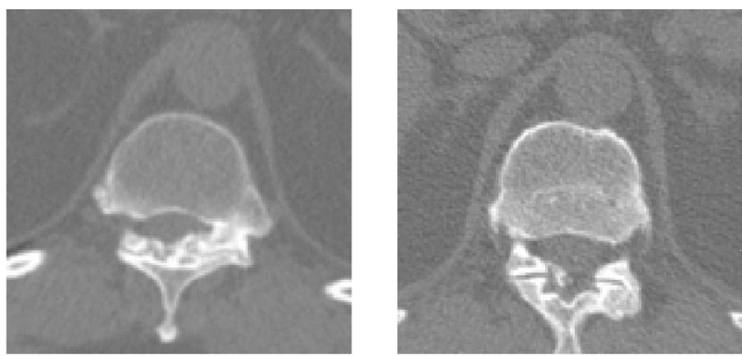

T12
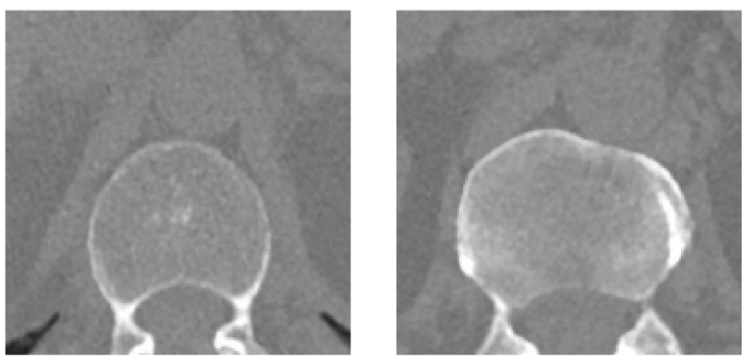

L1
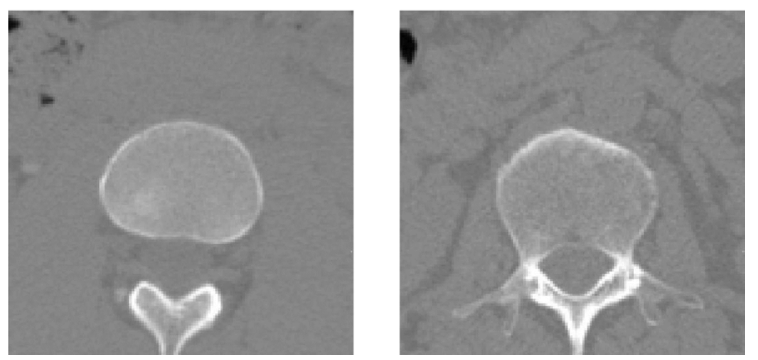

Figure 5 Examples of vertebral rims flattened by the aorta compared with not flattened rims at the same vertebral level. Each example was from a different subject.

tissue, may also be hypothesised. Periaortic fat secretes a variety of cytokines, chemokines, adipokines and growth factors that could potentially influence adjacent vertebrae. ${ }^{30} 31$ Most substances characterised from periaortic fat have been proinflammatory, and therefore might be thought to promote syndesmophyte development if diffusion to the area of the enthesis occurred. We did not find any association between aortic 
wall calcification and syndesmophyte distribution, suggesting that atheromatous inflammation in the adjacent aorta did not mediate this association, although proinflammatory mediators may be present in the absence of aortic calcification, and processes other than atherosclerosis may have paracrine effects on bone. However, to our knowledge, studies demonstrating that abnormalities in periaortic tissue can promote osteoproliferation have not been reported. Aortitis likely does not have a role, given its rarity and that it affects the aortic root and ascending aorta.

In contrast, the presence of vertebral flattening supports a mechanical hypothesis by demonstrating that the aorta is both close enough and powerful enough to shape vertebral morphology. ${ }^{22}$ In our study, flattening was most often seen at the thoracolumbar junction and when the aorta was close to the vertebrae. In the extreme, pressure from aortic aneurysms can cause extensive erosion of vertebral bodies. ${ }^{32} 33$ We hypothesise that aortic pulsation causes local movement of soft tissue to inhibit syndesmophyte formation in what may be the tissue-level analogue of exercise.

The strengths of this study include the use of a well-validated and sensitive measure of syndesmophytes, testing multiple IDSs, and examining associations with the distance to the aorta. The study also has some limitations. We studied a modest number of subjects; in particular, few were included in analyses of the mid-thoracic IDSs. However, the number was sufficient to detect strong associations with aortic distance. No subject had a rightside aorta to confirm an association with the aorta specifically, but the syndesmophyte deficit tracked with the position of the aorta down the spine and was not limited to the same angular sectors across IDSs or patients. Also, we did not directly investigate potential mechanisms that might mediate this association. Our goal was to investigate the interaction between syndesmophytes and the aorta and not to test a global model of syndesmophyte development.

Our results indicate that the aorta is associated with less frequent development of syndesmophytes in AS, possibly through mechanical effects. These findings suggest a complicated relationship between mechanical forces and syndesmophyte development, where forces with differing characteristics (location, directionality, intensity or constancy) may either promote or inhibit syndesmophyte growth. Skeletal tissues are mechanoresponsive and require loading to maintain normal structure. ${ }^{34} 35$ Mechanical stress that is supraphysiological or that affects inflamed tissues may initiate ectopic bone formation. ${ }^{93637}$ These findings, together with our results, suggest that the responses of bone to forces generated within the skeletal system (from muscle contraction or gravity) may differ from responses to extrinsic forces, as from the aorta. It is important to recognise that this influence is relative rather than absolute, as bridging syndesmophytes can encase the IDS, including the vertebral rim near the aorta. This indicates that the association between the aorta and syndesmophyte growth may be best characterised as a local slowing of growth. This local slowing may contribute to the low concordance between vertebral inflammation on MRI and future syndesmophytes, where syndesmophytes are not seen after 2 years in $78 \%-90 \%$ of anterior lumbar vertebral corners with inflammation. ${ }^{783839}$ Most importantly, our results indicate that factors other than vertebral inflammation may affect syndesmophyte development, including factors extrinsic to the spine.

Acknowledgements We thank Lori Guthrie, RN and Amanda Bertram for assistance.
Contributors MMW conceived the study. ST, JAF and MMW designed the study and $S T$ and $A D$ did the analysis. MMW drafted the manuscript and all authors provided critical review and approval of the final version.

Funding This work was supported by the Intramural Research Program, National Institute of Arthritis and Musculoskeletal and Skin Diseases, National Institutes of Health and by the Clinical Center, National Institutes of Health and the Johns Hopkins University School of Medicine General Clinical Research Center (grant number M01-RR00052 from the National Center for Research Resources/NIH)

Competing interests None declared.

Patient consent for publication Not required.

Provenance and peer review Not commissioned; externally peer reviewed.

Data availability statement Data will be available on request at the conclusion of the study.

\section{REFERENCES}

1 Jang JH, Ward MM, Rucker AN, et al. Ankylosing spondylitis: patterns of radiographic Involvement-A re-examination of accepted principles in a cohort of 769 patients. Radiology 2011;258:192-8.

2 Ramiro S, Stolwijk C, van Tubergen A, et al. Evolution of radiographic damage in ankylosing spondylitis: a 12 year prospective follow-up of the OASIS study. Ann Rheum Dis 2015;74:52-9.

3 Tan S, Yao J, Flynn JA, et al. Zygapophyseal joint fusion in ankylosing spondylitis assessed by computed tomography: associations with syndesmophytes and spinal motion. J Rheumatol 2017:44:1004-10.

4 Ball J. Enthesopathy of rheumatoid and ankylosing spondylitis. Ann Rheum Dis 1971:30:213-23.

5 Jevtic V, Kos-Golja M, Rozman B, et al. Marginal erosive discovertebral "Romanus" lesions in ankylosing spondylitis demonstrated by contrast enhanced Gd-DTPA magnetic resonance imaging. Skeletal Radiol 2000;29:27-33.

6 Appel H, Loddenkemper C, Grozdanovic Z, et al. Correlation of histopathological findings and magnetic resonance imaging in the spine of patients with ankylosing spondylitis. Arthritis Res Ther 2006;8.

7 Chiowchanwisawakit P, Lambert RGW, Conner-Spady B, et al. Focal fat lesions at vertebral corners on magnetic resonance imaging predict the development of new syndesmophytes in ankylosing spondylitis. Arthritis Rheumatol 2011;63:2215-25.

8 Baraliakos X, Heldmann F, Callhoff J, et al. Which spinal lesions are associated with new bone formation in patients with ankylosing spondylitis treated with anti-TNF agents? a long-term observational study using MRI and conventional radiography. Ann Rheum Dis 2014:73:1819-25.

9 Jacques $\mathrm{P}$, Lambrecht $\mathrm{S}$, Verheugen $\mathrm{E}$, et al. Proof of concept: enthesitis and new bone formation in spondyloarthritis are driven by mechanical strain and stromal cells. Ann Rheum Dis 2014;73:437-45

10 Ward MM, Reveille JD, Learch TJ, et al. Occupational physical activities and long-term functional and radiographic outcomes in patients with ankylosing spondylitis. Arthritis Rheum 2008;59:822-32.

11 Tan S, Dasgupta A, Yao J, et al. Spatial distribution of syndesmophytes along the vertebral rim in ankylosing spondylitis: preferential involvement of the posterolateral rim. Ann Rheum Dis 2016;75:1951-7.

12 Vernon-Roberts B, Pirie CJ, Trenwith V. Pathology of the dorsal spine in ankylosing hyperostosis. Ann Rheum Dis 1974;33:281-8.

13 Nathan H, Schwartz A. Inverted pattern of development of thoracic vertebral osteophytosis in situs inversus and in other instances of right descending aorta. Radiol Clin 1962;31:150-8.

14 Sebro R. Confirmation of the influence of descending aorta on osteophyte formation in dish. J Clin Rheumatol 2018;24:351-3.

15 Verlaan JJ, Westerveld LA, van Keulen JW, et al. Quantitative analysis of the anterolateral ossification mass in diffuse idiopathic skeletal hyperostosis of the thoracic spine. Eur Spine J 2011;20:1474-9.

16 van der Linden S, Valkenburg HA, Cats A. Evaluation of diagnostic criteria for ankylosing spondylitis. A proposal for modification of the New York criteria. Arthritis Rheum 1984;27:361-8.

17 MacKay K, Mack C, Brophy S, et al. The bath ankylosing spondylitis radiology index (BASRI): a new, validated approach to disease Assessment. Arthritis Rheum 1998;41:2263-70.

18 Tan S, Yao L, Ward MM. Thoracic syndesmophytes commonly occur in the absence of lumbar syndesmophytes in ankylosing spondylitis: a computed tomography study. J Rheumatol 2017:44:1828-32.

19 Tan S, Yao J, Flynn JA, et al. Quantitation of circumferential syndesmophyte height along the vertebral rim in ankylosing spondylitis using computed tomography. J Rheumatol 2015;42:472-8.

20 Tan S, Yao J, Flynn JA, et al. Quantitative measurement of syndesmophyte volume and height in ankylosing spondylitis using CT. Ann Rheum Dis 2014;73:544-50.

21 Sarwahi V, Gecelter RC, Wendolowski SF, et al. CT-based anatomical evaluation of prevertebral structures with respect to vertebral body using a clock-face analogy. SPINE 2015:40:1918-25 
22 Taylor JR. Scoliosis and Growth: Patterns of Asymmetry in Normal Vertebral Growth. Acta Orthop Scand 1983:54:596-602.

23 Cramer GD. The thoracic spine. In: Cramer GD, Darby SA, eds. Basic and clinical anatomy of the spine, spinal cord, and ANS. 2nd edn. St. Louis: Mosby Inc 2005: 210-41

24 Culver GJ, Pirson HS. Preventive effect of aortic pulsations on osteophyte formation in the thoracic spine. Am J Roentgenol Radium Ther Nucl Med 1960;84:937-40.

25 Nathan H. Osteophytes of the vertebral column. An anatomic study of their development according to age, race, and sex with considerations as to their etiology and significance. J Bone Joint Surg 1962;44-A:243-68.

26 Nathan H. Osteophytes of the spine compressing the sympathetic trunk and splanchnic nerves in the thorax. Spine 1987;12:527-32

27 Watanabe S, Terazawa K. Age estimation from the degree of osteophyte formation of vertebral columns in Japanese. Leg Med 2006;8:156-60.

28 Goldberg RP, Carter BL. Absence of thoracic osteophytosis in the area adjacent to the aorta: computed tomography demonstration. J Comput Assist Tomogr 1978;2:173-5.

29 Appel H, Maier R, Loddenkemper C, et al. Immunohistochemical analysis of osteoblasts in zygapophyseal joints of patients with ankylosing spondylitis reveal repair mechanisms similar to osteoarthritis. J Rheumato/ 2010;37:823-8.

30 Thalmann S, Meier C. Local adipose tissue depots as cardiovascular risk factors. Cardiovasc Res 2007;75:690-701.

31 G. Spiroglou S, G. Kostopoulos C, N. Varakis J, et al. Adipokines in periaortic and epicardial adipose tissue: differential expression and relation to atherosclerosis. JAT 2010;17:115-30.
32 Hertzer NR. Aneurysms with backbone - an inside view of vertebral erosion. Ann Vasc Surg 2014;28:1813-5.

33 Wansink J, van der Kleij FGH. Vertebral-Body erosion in thoracic aortic aneurysm. N Engl J Med 2016;374:e10

34 Rosa N, Simoes R, Magalhães FD, et al. From mechanical stimulus to bone formation: a review. Med Eng Phys 2015;37:719-28.

35 Sun HB, Schaniel C, Leong DJ, et al. Biology and mechano-response of tendon cells: progress overview and perspectives. J Orthop Res 2015;33:785-92.

36 Tsukamoto N, Maeda T, Miura H, et al. Repetitive tensile stress to rat caudal vertebrae inducing cartilage formation in the spinal ligaments: a possible role of mechanical stress in the development of ossification of the spinal ligaments. J Neurosurg Spine 2006;5:234-42.

37 Sherlock JP, Joyce-Shaikh B, Turner SP, et al. IL-23 induces spondyloarthropathy by acting on ROR- $\gamma t+C D 3+C D 4-C D 8-$ entheseal resident T cells. Nat Med 2012;18:1069-76.

38 Maksymowych WP, Chiowchanwisawakit P, Clare T, et al. Inflammatory lesions of the spine on magnetic resonance imaging predict the development of new syndesmophytes in ankylosing spondylitis: evidence of a relationship between inflammation and new bone formation. Arthritis Rheum 2009;60:93-102.

39 van der Heijde D, Machado P, Braun J, et al. MRI inflammation at the vertebral unit only marginally predicts new syndesmophyte formation: a multilevel analysis in patients with ankylosing spondylitis. Ann Rheum Dis 2012;71:369-73. 